\title{
Tension Type Headache in Adolescence and Childhood: Where Are We Now?
}

\author{
Teshamae S. Monteith • Till Sprenger
}

Published online: 7 October 2010

(C) The Author(s) 2010. This article is published with open access at Springerlink.com

\begin{abstract}
Tension type headache (TTH) is a primary headache disorder considered common in children and adolescents. It remains debatable whether TTH and migraine are separate biological entities. This review summarizes the most recent literature of TTH with regards to children and adolescents. Further studies of TTH are needed to develop a biologically based classification system that may be facilitated through understanding changes in the developing brain during childhood and adolescence.
\end{abstract}

Keywords Tension-type headache · Migraine $\cdot$ Children . Adolescents $\cdot$ Calcitonin gene receptor peptide .

Diagnostic criteria

\section{Introduction}

Primary headaches are common in children and adolescents. Tension-type headache (TTH) and migraine are important sources of disability with several health-related considerations. In comparison to migraine, little is known about TTH in children; most of our knowledge has been extrapolated from adult studies. Childhood and adolescence mark periods of rapid growth, emotional maturation, physiological perturbations, and hormonal changes, all

T. S. Monteith $(\bowtie)$

UCSF Headache Center,

1701 Divisadero Street, Suite 480,

San Francisco, CA 94115, USA

e-mail: teshamae.monteith@ucsf.edu

\section{T. Sprenger}

Neurologische Klinik und Poliklinik, Klinikum rechts der Isar,

Technische Universität München,

Ismaninger Strasse 22,

81675 Munich, Germany which may influence the expression of primary headache disorders in those with a predisposition [1]. For example, the prevalence of migraine increases with age in both males and females during preadolescence [2]. With puberty, there is a higher prevalence of migraine in girls than boys, suggesting a role of female sex hormones in the expression of headache. The prevalence of TTH also increases with age in adolescents [3•]. It is important to better understand TTH in this age group because these changes influence headache phenotypes and may provide important points of considerations when distinguishing TTH from migraine.

The diagnoses of TTH and migraine are typically based on the second edition of the International Headache Society's International Classification of Headache Disorders (ICHD-II) [4]. According to the criteria, TTH lasts from $30 \mathrm{~min}$ to 7 days and is classified as a bilateral pressure, tightening or nonpulsating headache of mild to moderate intensity, occurring without aggravation by routine physical exertion and without nausea or vomiting, but may have either photophobia or phonophobia but not both (Table 1) [4]. It may be argued that the classification for TTH lacks sensitivity and specificity because the diagnosis of TTH is essentially made by the lack of associated symptoms diagnostic of migraine. The addition of photophobia and phonophobia in TTH diagnosis may lead to a diagnosis of TTH in quite a few cases that may be better classified as migraine.

Diagnosis of TTH in children has obvious limitations. There are difficulties related to the challenges in taking histories in young children. In one retrospective study testing the ICHD-II diagnoses of migraine and TTH in children, many children were unclassifiable because children were not able to communicate information regarding pain quality, pain score, and aggravation by movement or routine activities [5]. Behavior may be a more useful 
Table 1 ICHD-II Criteria for migraine without aura and tension-type headache for children
ICHD-II The International Classification of Headache Disorders, second edition

(Data from the Headache Classification Subcommittee of the International Headache Society [4].)
Migraine without aura (code 1.1)
A. At least five attacks fulfilling criteria B-D
B. Attacks lasting 1-72 h (untreated or successfully treated)
C. Two of the following characteristics:
1. Migraine headache commonly is bilateral; occipital headache in children is rare
2. Pulsating quality
3. Moderate to severe intensity
4. Aggravated by, or causing avoidance of, routine physical activity
D. At least one of the following associated symptoms:
1. Nausea and/or vomiting
2. Photophobia and phonophobia (photophobia may be inferred from behavior in young children) Subtypes: Episodic ( $<15$ days/mo) and chronic $(\geq 15$ days/mo on average for $>3$ mo)
Tension-type headache (code 2 )
A. At least 10 episodes occurring on $<1$ day/mo on average (12 days/y) and fulfilling criteria B-D
B. Headache lasting $30 \mathrm{~min}$ to 7 days
C. Two of the following characteristics:
1. Bilateral location
2. Pressing/tightening (nonpulsating) quality
3. Mild to moderate intensity
4. Not aggravated by routine activity
D. At least one of the following associated symptoms:
1. No nausea or vomiting
2. Photophobia or phonophobia
Subtypes
1. Episodic
A. May occur with or without pericranial muscle tenderness
1. Infrequent
a. At least 10 episodes occurring on $<1$ day/mo on average ( $<12$ days/y) and fulfilling criteria B-D
2. Frequent
a. At least 10 episodes occurring on $\geq 1$ but $<15$ day/mo for at least 3 months
2. Chronic
A. $\geq 15$ days $/$ mo on average for $>3 \mathrm{mo}$

measure of pain intensity than pain scores in younger children. Clues to look for include a reduction in social interaction or play, a child that once was active that later becomes sedentary and requires more sleep, or perhaps a child that appears less involved in school. Altogether, studies aimed at validating the ICHD-II diagnostic criteria for TTH on a phenotypicalbiological basis are necessary to adequately interpret current studies and begin to answer the pressing questions that exist [6]. This review aims to summarize the current studies pertaining to TTH in the pediatric and adolescence age groups, the applicability of the ICHD-II, and clinical and pathophysiological considerations.

\section{Classification, Phenotype, and Diagnostic Challenges}

The classification of TTH is based on its phenotype as defined by the ICHD-II [4]. According to ICHD-II, TTH generally is defined as a bilateral headache with pressing or band-like quality of low to moderate intensity. TTH can be distinguished further in terms of frequency, which can vary in individuals: the infrequent episodic subtype occurs less than once monthly, the frequent episodic subtype occurs on less than 15 days per month, and the chronic subtype occurs 15 days or more per month for over 3 months. The distinctions based on frequencies are rather arbitrary and the chosen terminology, such as the context in which the word chronic is used, can be confusing. Moreover, the high prevalence and the variance over time for infrequent and frequent TTH make genetic studies harder to interpret and distinguish from chance [7].

Migraine can commonly present as a bilateral, shortlasting headache, with episode-to-episode differences in associated features resembling TTH. Another problem is that the phenotype may not be fully developed with age because the phenotype of migraine without aura can evolve 
in adolescence or early adulthood from a bilateral to unilateral headache [4]. Ultimately, the variability in phenomenology raises the possibility that, at least in some patients, we may be speaking of the same disorder with both typical and atypical attacks of migraine [6,7].

In clinical practice, it is not always easy to distinguish between the two entities (Fig. 1). Altogether, it has been debated whether such distinctions need to be made because there seem to be more similarities than differences [8]. Due to several considerations, a significant number of patients cannot be diagnosed, remain unclassifiable, or carry the diagnosis of probable migraine or probable TTH [9]. For migraine in children, the ICHD-II recognizes that attacks may be of shorter duration, lasting from 1 to $72 \mathrm{~h}$, with bilateral location and pain intensities that may be inferred from their behavior. According to one study analyzing the ICHD-II diagnosis of migraine and TTH in children, the criteria classify TTH and migraine well, although many patients were left unclassifiable with a reduction in duration not making a significant difference [9]. The authors suggested that the duration criteria be eliminated for TTH and migraine because a significant increase in duration has been shown to occur with age. Mixed phenotypes propose yet another diagnostic issue. In one study, the prevalence of coexisting TTH and migraine was $6 \%$ based on a population-based study in Swedish schoolchildren [10]. This certainly can be supported by Lipton et al. [11], who showed that TTH in patients with migraine responds to sumatriptan whereas patients with TTH alone do not, supporting the continuum theory at least in patients with both disorders.

For chronic forms, problems also exist when children can be diagnosed with both chronic TTH (CTTH) and chronic migraine. It has been argued that these patients' ailments could be diagnosed as chronic migraine based on the new appendix criteria for a broader concept of chronic migraine [12]. A retrospective chart review of chronic daily headache in French children and adolescents supports the use of detailed histories and diaries along with the

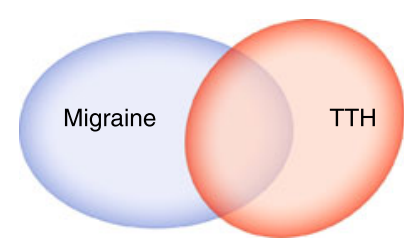

Fig. 1 Diagnostic ambiguity of current criteria for migraine and TTH in pediatric and adolescent populations. Difficulties in diagnosing TTH in children from migraine include 1) frequent headache transformations; 2) changes in phenotype with age and sex; 3) coexisting migraine and TTH; 4) communication barriers; 5) not fulfilling the prior episode or duration requirement; 6) methodological variability in epidemiological studies; and 7) lack of a biologicalbased classification system for TTH and migraine
Silberstein-Lipton criteria for chronic daily headache, which encompasses transformed migraine, CTTH, and new daily-persistent headache. The ICHD-II revised criteria also were found useful and allowed for the diagnosis of chronic migraine, new daily-persistent headache, CTTH, CTTH and migraine without aura, CTTH and migraine with and without aura, probable CTTH, and CTTH with probable migraine without aura.

When the phenotype is unclassifiable, it may be helpful to ascertain further information to determine a diagnosis. Parents usually accompany their children and make it plausible to obtain a detailed family history of headache. Classically, TTH lacks the circadian rhythmicity seen in other primary headache disorders such as migraine or cluster headache. Ice cream headaches and motion sickness in the childhood history of the patient may be useful markers for migraine in unclear diagnostic circumstances. A history of cyclical vomiting or recurrent abdominal pain in an unclassifiable patient is suggestive of an evolution to migraine. Recurrence may be reassuring in confirming the benign nature of a headache disorder. On the other hand, the number of episodes in young children is limited by a short lifespan, and therefore, it has been suggested to eliminate the number of episodes in young children. This also may be reasonable keeping in mind headache transformation in this age group.

High rates of headache transformation between TTH and migraine create diagnostic challenges. For example, a 4-year annual follow up study in 12- to 17-year-old patients with headache showed that headache diagnosis changed in $20.69 \%$ of patients on follow-up [13]. Specifically, there was transformation of headache type in 4 of 50 patients with migraine (8\%), 10 of 24 patients with TTH (41\%), and 4 of 13 patients with other headache types $(30.8 \%)$. Headache transformation suggests the possibility of a common underlying biology or coinheritance of TTH and migraine.

\section{Neurobiological Considerations}

The evidence for the existence of TTH as a separate biological disorder needs further validation. Nitric oxide (NO) appears to be an important molecule involved in regulation of cerebral and extracerebral blood flow, regulation or arterial diameter, and nociceptive signaling possibly linked to release of calcitonin gene-related peptide (CGRP). Nitroglycerin infusion has been shown to trigger immediate and delayed headaches in patients with either migraine or CTTH, and the authors suggested that NO-related central sensitization may be an important common denominator in pain mechanisms of TTH and migraine [14]. While nociception from myofascial tissues is considered important in TTH, the role of a peripheral mechanism as an inciting 
factor is not clearly known, and certainly central factors are important for CTTH because general hypersensitivity to pain stimuli has been demonstrated [15]. It has been suggested that peripheral inputs also are important in migraine pathophysiology, although the presences of aura, premonitory symptoms, and functional imaging studies support central activation as a primary mechanism [16, 17]. In a magnetic resonance imaging voxel-based morphometry study comparing 20 patients with CTTH and 20 patients with medication overuse headache with 40 controls patients, only patients with CTTH demonstrated significant gray matter decreases in areas of the brain involved in nociceptive transmission [18]. Such changes seem to be reversible, as shown in other chronic pain conditions [19].

Further subclassification for the episodic and chronic subtypes of TTH can be made for children with or without pericranial tenderness, caused from excessive contraction of the jaw or scalp muscles [4]. In a blinded study, restricted neck mobility was significant in children with CTTH with pericranial tenderness for flexion, extension, and right-left lateral rotation [20]. Another study showed increased pericranial muscle tenderness at palpation in children with migraine, but failed to find an association in the neckshoulder regions of children with TTH [21]. The study found no differences in mean pressure pain thresholds between children in the TTH, migraine, and nonheadache groups. Certainly, neck pain is a common finding in migraine, associated with activation of the trigeminocervical complex. Altogether, muscle factors may be more important in the pathogenesis of TTH than migraine, but they are certainly seen in both disorders, and are not of diagnostic utility [22].

Migraine is considered a neurobiological disorder with a susceptibility to attacks based on external and internal triggers. Some studies have indicated that the triggering factors for migraine are greater than for TTH in this population $[3 \bullet, 23]$. Stress and peripheral factors such as posture are considered important in TTH. In an epidemiologically based population study, precipitation by sleeplessness, sunlight, hunger, exercise, and watching TV were more common in the migraine group than children with TTH. The reduced triggerability of TTH has been argued as a way to distinguish the two entities, and it has been suggested that lack of triggerability of TTH should become a part of the ICHD-III criteria [5]. Although helpful in some cases, this would be an arbitrary distinction.

CGRP levels are not significantly elevated during TTH in adults [24], unlike migraine, where CGRP levels were predictive of pediatric migraine when compared to the nonmigraine group [25]. The CGRP levels were significantly elevated when comparing levels during an attack versus out of an attack. CGRP has been implicated in the pathophysiology of migraine. CGRP is a neuropeptide produced by alternative RNA splicing of the calcitonin gene. CGRP receptors are found on meningeal blood vessels, trigeminal ganglion and sensory afferents, in the periaqueductal gray, and in other areas of the brain associated with migraine pathogenesis [26]. Stimulation of the trigeminal ganglion can result in elevations of CGRP and substance P [27, 28]; however, only CGRP was significantly elevated in the external jugular veins in patients during an acute attack of migraine. Elevated CGRP levels are associated with severe and prolonged headaches, and the headache can be normalized with sumatriptan treatment [28, 29]. Therefore, one caution in interpreting these results is that CGRP elevation is associated with headache severity, so that it may be an artifact of our classification system and, in line with the continuum theory, that nonmigraine or TTH is not associated with significantly elevated levels during acute attacks [24]. There is a relative over-representation of CGRP-enriched afferents related to dural innervation, which may explain the importance of CGRP in migraine and the trigeminal autonomic cephalgias [30]. It is arguable whether this is at all important for TTH because headache perception in TTH may be explained by referred pain from trigger points in the craniocervical muscles [31]. Further work that aims to identify biomarkers that may distinguish migraine from TTH is necessary to add biological validity to our classification system.

\section{Epidemiology}

The variability in the prevalence estimates of TTH has been attributed to environmental and genetic factors. According to estimates based on several prevalence studies, TTH affects about $31 \%(10 \%-72 \%)$ of children [32]. In one study, a family history of headache was found in $40 \%$ of patients with TTH, and first-degree relatives had a 2.1- to 3.9-fold increased risk of CTTH compared to the general population $[33,34]$. In another study, $46 \%$ of patients with migraine were found to have a family history of migraine as compared to $18 \%$ of patients with migraine having a family history of TTH [35]. On the other hand, one study, a population-based study of 33,764 adolescent and adult twins, showed that the risk and frequency of TTH was found to be higher in patients with migraine, perhaps because they carry a single diagnosis of migraine [7]. Altogether, family histories are limited in that these disorders have not been fully defined.

Age is a risk factor for TTH in children and adolescents similar to migraine. According to a study of Swedish schoolchildren between the ages of 7 and 15 years, both TTH and migraine increased with age, especially in girls [10]. The influence of age also was seen in one study, conducted in a pediatric specialty care center, where age 
accounted for more variance than sex in headache severity, duration, frequency, and disability [36]. In a 6-year epidemiological follow-up study in children, headache was reported in 909 of 1,155 adolescents and commonly changed; $57.5 \%$ of headaches were diagnosed as TTH, $18.6 \%$ were diagnosed as migraine, and $2.6 \%$ were unspecified [3•]. The most important variation was the increase in TTH with medication overuse occurring frequently in $8.7 \%$ of adolescents with TTH.

Several large headache global initiatives are underway to determine the prevalence and impact of headache disorders. The "Lifting the Burden" campaign is a World Health Organization global initiative to assess the prevalence and burden of headache. Based on the initiative, the prevalence data on pediatric TTH is relatively sparse, with estimates for Norway, Sweden, and Brazil of $23.2 \%, 11.8 \%$, and $72.3 \%$, respectively [32]. Winkler et al. [37•] studied the prevalence of headache in rural Tanzania through a door-todoor survey and found an exceedingly low prevalence rate for TTH. The average 1-year prevalence of TTH in the age group of 0 to 10 years was $0.04 \%$ based on the ICHD-II criteria. Between the ages of 11 and 20 years, the average 1 -year prevalence was only $1.3 \%$. Further efforts that uncover the scale, the impact, and the factors associated with population differences of TTH in younger generations need to be made on the local, national, and global levels.

The discrepancies in prevalence have been attributed to differences in methodology including prevalence estimates, usage of the ICHD-II versus other diagnostic criteria, and interview types. While multiple headache diagnoses can be appreciated in a clinic setting through a clinical interview, questionnaire studies may be sensitive to more severe phenotypes, resulting in single diagnoses of migraine. Other considerations that may account for population differences are psychosocial and cultural factors of pain reporting. The Eurolight project is an initiative with the objective of gathering updated reliable comparable information regarding migraine, TTH, and chronic headache in selected European countries [38]. Both systematic and consistent methodological approaches are necessary to determine the true prevalence of TTH worldwide.

Recently, epidemiological studies have focused on determining the population dynamics of headache based on frequencies. A transitional model of TTH shows that low-frequency TTH may remit or transition to highfrequency TTH, which may in turn remit or transition to CTTH [39]. This may be an overly simplified approach, as there is a substantial rate of transformation between migraine and TTH in follow-up studies. Even so, epidemiological studies assessing headache frequency have the advantage of estimating primary headache without the limitations of ICHD-II, such as those subjects that are unable to be diagnosed. In a population-based study of preadolescent school children in Brazil, the prevalences of high-frequency headache and chronic daily headache were $2.5 \%$ and $1.6 \%$, respectively [39]. High frequency is an important risk factor for chronic daily headache in adults and is important to consider in children.

Chronic daily headache is important to study in this age group, as in one clinic based study, early onset disease was associated with a worst outcome [40]. CTTH was more common in adolescents than in adults, but medication overuse was not seen.

\section{Management}

Headache can have a significant impact on the lives of children and parents; however, there may be too much attention to psychosocial variables, which are more consistent with the older terminology of tension headache as opposed to TTH. For example, TTH is associated with fewer close friends and had a higher rate of divorced parents [41]. Another study assessing the cognitive profiles of children with headache when compared to control patients, showed a significant negative correlation between total intelligence quotient, performance intelligence quotient, and the frequency of attacks [42]. This indicates that children with frequent headache perform not as well as they potentially could in terms of cognition because of the headaches. This is a strong argument for treatment. Perhaps these studies should be approached agnostically to avoid errors of attribution, especially for a disorder that is so common.

A recent population-based cross-section study of psychopathological symptoms, such as emotional symptoms, conduct problems, hyperactivity/inattention, and peer problems, in adolescents with headache did not find a significantly increased risk in patients with TTH; however, if psychological factors are present, they should be appropriately managed in parallel [43].

More controlled studies are needed to assess the efficacy of nonpharmacological approaches. In an uncontrolled study, electromyographic biofeedback-assisted relaxation techniques appeared effective in reducing headaches in a 3-year juvenile TTH follow-up study [44]. More studies for physical therapy are needed; a study to determine the effects of specific strength training versus lifestyle counseling in children with TTH currently is underway (NCT01155557) [45]. A study of diet and lifestyle in high school students suggests different habits in regular intake of breakfast, physical activity, and consumption of alcohol in adolescents with any type of headache may be important [46]. Behavioral approaches have the benefit of minimizing the need for pharmaceuticals and their potential side effects; however, trigger management and lifestyle modifications may be challenging due to issues of adherence. 
The European Federation of Neurological Societies guidelines for treatment of TTH make general recommendations, which can be applied to children [47]. Simple analgesics and NSAIDs are first-line agents considered effective. The evidence for the efficacy of prophylaxis is limited; amitriptyline often is used, as in migraine, although more randomized controlled trials are needed [48]. Drugs blocking NO production may be effective for CTTH and deserve further exploration [49]. Ultimately, one important aspect of studies in this age group is the issue of expectation or high placebo rates, which can be accounted for with a crossover design. On the other hand, the high placebo rate may be used therapeutically.

\section{Conclusions}

To date, little is understood about the biological nature of TTH in children and adolescents. Growth and development with age are associated with many changes influencing the phenotypic expression of headache. Headache transformation in young children and adolescents is common, suggesting the possibility of similar biology at least in some patients. Prevalence estimates are limited by differences in methodological approaches including variability in diagnostic criteria. Studies based on identifying risk factors for frequent headache may better to identify at risk populations for TTH and migraine. Altogether, face-to-face structured interviews are optimal because longer histories of migraines over time or "chronicity" may trend to more featureless CTTH $[50,51]$.

Future research is necessary to validate upcoming classifications of migraine and TTH, especially in children and adolescents. Ultimately, we await the point in our scientific understanding where TTH can be diagnosed beyond its clinical phenotype because the current classification leaves many children unclassifiable. It also is with great anticipation that models of head pain, identifications of biomarkers, or advances in imaging pave the way to a more biologically accurate diagnostic classification system. With these goals in mind, we will be on our way to ascertain the true impact of the disorder, develop the best treatments, and effectively reduce disability.

Acknowledgements The authors would like to thank Dr. Peter J. Goadsby and Dr. Neil Raskin for their teaching and thoughts on migraine and tension-type headache.

Disclosures No potential conflicts of interest related to this article were reported.
Open Access This article is distributed under the terms of the Creative Commons Attribution Noncommercial License which permits any noncommercial use, distribution, and reproduction in any medium, provided the original author(s) and source are credited.

\section{References}

Papers of particular interest, published recently, have been highlighted as:

- Of importance

1. Aegidius KL, Zwart JA, Hagen K, et al.: Increased headache prevalence in female adolescents and adult women with early menarche. The Head-HUNT Studies. Eur J Neurol 2010 Jul 15 (Epub ahead of print).

2. Lipton RB, Bigal ME, Diamond M, et al.: Migraine prevalence, disease burden, and the need for preventive therapy. Neurology 2007, 68:343-349.

3. • Ozge A, Sasmaz T, Cakmak SE, et al.: Epidemiological-based childhood headache natural history study: After an interval of six years. Cephalalgia 2010, 30:703-712. The authors propose that there are significant changes in headache phenotypes in children.

4. Headache Classification Subcommittee of the International Headache Society: The International Classification of Headache Disorders: 2nd edition. Cephalalgia 2004, 24(Suppl 1):9160

5. Rossi LN, Cortinovis I, Menegazzo L, et al.: Classification criteria and distinction between migraine and tension-type headache in children. Dev Med Child Neurol 2001, 43:45-51.

6. Goadsby P: Chronic tension-type headache. Clin Evid 2002, (7):1145-1152.

7. Russell MB, Levi N, Saltyte-Benth J, Fenger K: Tension-type headache in adolescents and adults: a population based study of 33,764 twins. Eur J Epidemiol 2006, 21:153-160.

8. Cady R, Schreiber C, Farmer K, Sheftell F: Primary headaches: a convergence hypothesis. Headache 2002, 42:204-216.

9. Rossi LN, Vajani S, Cortinovis I, et al.: Analysis of the International Classification of Headache Disorders for diagnosis of migraine and tension-type headache in children. Dev Med Child Neurol 2008, 50:305-310.

10. Laurell K, Larsson B, Eeg-Olofsson O: Prevalence of headache in Swedish schoolchildren, with a focus on tension-type headache. Cephalalgia 2004, 24:380-388.

11. Lipton RB, Stewart WF, Cady R, et al.: 2000 Wolfe Award. Sumatriptan for the range of headaches in migraine sufferers: results of the Spectrum Study. Headache 2000, 40:783-791.

12. Olesen J, Bousser MG, Diener HC, et al.: New appendix criteria open for a broader concept of chronic migraine. Cephalalgia 2006, 26:742-746.

13. Karli N, Bican A, Zarifoğlu M: Course of adolescent headache: 4-year annual face-to-face follow-up study. J Headache Pain 2010, 11:327-334.

14. Ashina M, Bendtsen L, Jensen R, Olesen J: Nitric oxide-induced headache in patients with chronic tension-type headache. Brain 2000, 123:1830-1837.

15. Bendtsen L, Jensen R, Olesen J: Decreased pain detection and tolerance thresholds in chronic tension-type headache. Arch Neurol 1996, 53:373-376.

16. Afridi SK, Matharu MS, Lee L, et al.: A PET study exploring the laterality of brainstem activation in migraine using glyceryl trinitrate. Brain 2005, 128:932-939. 
17. Weiller C, May A, Limmroth V, et al.: Brain stem activation in spontaneous human migraine attacks. Nat Med 1995, 1:658-660.

18. Schmidt-Wilcke T, Leinisch E, Straube A, et al.: Gray matter decrease in patients with chronic tension type headache. Neurology 2005, 65:1483-1486.

19. Rodriguez-Raecke R, Niemeier A, Ihle K, et al.: Brain gray matter decrease in chronic pain is the consequence and not the cause of pain. J Neurosci 2009, 29:13746-13750.

20. Fernández-Mayoralas DM, Fernández-de-Las-Peñas C, PalaciosCeña $\mathrm{D}$, et al.: Restricted neck mobility in children with chronic tension type headache: a blinded, controlled study. J Headache Pain 2010 May 28 (Epub ahead of print).

21. Attila P, Metsähonkala L, Mikkelsson M, et al.: Muscle tenderness in pericranial and neck-shoulder region in children with headache. A controlled study. Cephalalgia 2002, 22:340-344.

22. Oksanen A, Pöyhönen T, Metsähonkala L, et al.: Neck flexor muscle fatigue in adolescents with headache: an electromyographic study. Eur J Pain 2007, 11:764-772.

23. deGrauw TJ, Hershey AD, Powers SW, Bentti AL: Diagnosis of migraine in children attending a pediatric headache clinic. Headache 1999, 39:481-485.

24. Ashina M: Calcitonin gene-related peptide in tension-type headache. ScientificWorldJournal 2002, 2:1527-1531.

25. Fan PC, Kuo PH, Chang SH, et al.: Plasma calcitonin gene-related peptide in diagnosing and predicting paediatric migraine. Cephalalgia 2009, 29:883-890.

26. Arulmani U, Maassenvandenbrink A, Villalón CM, Saxena PR: Calcitonin gene-related peptide and its role in migraine pathophysiology. Eur J Pharmacol 2004, 500:315-330.

27. Goadsby PJ, Edvinsson L, Ekman R: Vasoactive peptide release in the extracerebral circulation of humans during migraine headache. Ann Neurol 1990, 28:183-187.

28. Goadsby PJ, Edvinsson L: The trigeminovascular system and migraine: studies characterizing cerebrovascular and neuropeptide changes seen in humans and cats. Ann Neurol 1993, 33:48-56.

29. Goadsby PJ: Calcitonin gene-related peptide antagonists as treatments of migraine and other primary headaches. Drugs 2005, 65:2557-2567.

30. O'Connor TP, van der Kooy D: Enrichment of a vasoactive neuropeptide (calcitonin gene related peptide) in the trigeminal sensory projection to the intracranial arteries. J Neurosci 1988, 8:2468-2476.

31. Fernández-de-las-Peñas C, Schoenen J: Chronic tension-type headache: what is new? Curr Opin Neurol 2009, 22:254-261.

32. Stovner LJ, Hagen K, Jensen R, et al.: The global burden of headache: a documentation of headache prevalence and disability worldwide. Cephalalgia 2007, 27:193-210.

33. Russell MB, Ostergaard S, Bendtsen L, Olesen J: Familial occurrence of chronic tension-type headache. Cephalalgia 1999, 19:207-210.

34. Friedman AP, von Storch TJ, Merritt HH: Migraine and tension headaches: a clinical study of two thousand cases. Neurology 1954, 4:773-788.

35. Lance JW, Anthony M: Some clinical aspects of migraine. A prospective survey of 500 patients. Arch Neurol 1966, 15:356-361.
36. Slater S, Crawford MJ, Kabbouche MA, et al.: Effects of gender and age on paediatric headache. Cephalalgia 2009, 29:969-973.

37. - Winkler A, Stelzhammer B, Kerschbaumsteiner K, et al.: The prevalence of headache with emphasis on tension-type headache in rural Tanzania: a community-based study. Cephalalgia 2009, 29:1317-1325. According to the authors, age is an important factor associated with the phenotype of pediatric headache.

38. Stovner LJ, Andree C: Prevalence of headache in Europe: a review for the Eurolight project. J Headache Pain 2010, 11:289299.

39. Arruda MA, Guidetti V, Galli F, et al.: Frequent headaches in the preadolescent pediatric population: a population-based study. Neurology 2010, 74:903-908.

40. Bigal ME, Lipton RB, Tepper SJ, et al.: Primary chronic daily headache and its subtypes in adolescents and adults. Neurology 2004, 63:843-847.

41. Karwautz A, Wöber C, Lang T, et al.: Psychosocial factors in children and adolescents with migraine and tension-type headache: a controlled study and review of the literature. Cephalalgia 1999, 19:32-43.

42. Parisi P, Verrotti A, Paolino MC, et al.: Headache and cognitive profile in children: a cross-sectional controlled study. J Headache Pain 2010, 11:45-51.

43. Milde-Busch A, Boneberger A, Heinrich S, et al.: Higher prevalence of psychopathological symptoms in adolescents with headache. A population-based cross-sectional study. Headache 2010, 50:738-748.

44. Grazzi L, Andrasik F, D'Amico D, et al.: Electromyographic biofeedback-assisted relaxation training in juvenile episodic tension-type headache: clinical outcome at three-year follow-up. Cephalalgia 2001, 21:798-803.

45. ClinicalTrials.gov: The Effect of Specific Strength-Training on Tension-Type Headache in Children 9-17 Years. Available at http:// clinicaltrials.gov/ct2/show/NCT01155557. Accessed September 2010.

46. Milde-Busch A, von Kries R, Thomas S, et al.: The association between use of electronic media and prevalence of headache in adolescents: results from a population-based cross-sectional study. BMC Neurol 2010, 10:12.

47. Bendtsen L, Evers S, Linde M, et al.: EFNS guideline on the treatment of tension-type headache: report of an EFNS task force. Eur J Neurol 2010 May 11 (Epub ahead of print).

48. Lance JW, Curran DA: Treatment of chronic tension headache. Lancet 1964, 1:1236-1239.

49. Olesen $\mathrm{J}$ : The role of nitric oxide (NO) in migraine, tension-type headache and cluster headache. Pharmacol Ther 2008, 120:157171.

50. Cuvellier JC, Couttenier F, Auvin S, Vallée L: The classification of chronic daily headache in French children and adolescents: A comparison between the second edition of the International Classification of Headache Disorders and Silberstein-Lipton criteria. Neuropsychiatr Dis Treat 2008, 4:263-267.

51. Silberstein SD, Lipton RB, Sliwinski M: Classification of daily and near-daily headaches: field trial of revised IHS criteria. Neurology 1996, 47:871-875. 